\title{
TITLE: Fast 3D Ultrashort echo-time spiral projection imaging using Golden-angle: A flexible protocol for in vivo mouse imaging at high magnetic field.
}

Authors : Charles R Castets1,2, William Lefrançois1,2, Didier Wecker3, Emeline J Ribot1,2, Aurélien J Trotier1,2, Eric Thiaudière1,2, Jean-Michel Franconi1,2 and Sylvain Miraux 1,2*

\section{INSTITUTION}

1 - Centre de Resonance Magnetique des Systèmes Biologiques, UMR5536 CNRS, Bordeaux, France

2 - Centre de Resonance Magnetique des Systèmes Biologiques, UMR5536 Université de Bordeaux, Bordeaux, France

3 - Bruker Biospin MRI GMBH, Ettlingen, Germany

*Corresponding author: Sylvain Miraux Centre de Resonance Magnetique des Systèmes Biologiques, UMR5536 CNRS, Bordeaux, France 146 rue Léo Saignat 33076 Bordeaux Cedex miraux@rmsb.u-bordeaux2.fr

\section{WORD COUNT: 3619}

\section{Running Title: Golden-angle Spiral projection imaging}

KEYWORDS: Spiral, 3D, UTE, golden-angle, 7T, self-gating, mouse. 


\begin{abstract}
Purpose: To develop a fast 3D k-space encoding method based on spiral projection imaging coupled with an interleaved golden-angle approach and to validate it for angiography on small animal models.
\end{abstract}

Methods: A disk-like trajectory, where each disk contained numerous spirals enabling ultra-short echo time was developed. The $3 \mathrm{D}$ encoding was performed by tilting the disks with a golden angle. The point spread function and sharpness were measured at different $\mathrm{T} 2 *$ values using variable undersampling ratio. Then, the sampling method was validated on whole brain time-of-flight angiography and USPIO enhanced free-breathing liver angiography in mice at 7T.

Results: In vitro results demonstrated the robustness of the method for short T2* and high undersampling ratio. In vivo experiments showed the ability to properly detect small vessels in the brain with acquisition time shorter than $1 \mathrm{~min}$. The insensitivity of this protocol for motion and flow artifacts was demonstrated on free-breathing mice liver angiography.

Conclusion: The method proposed here allowed to perform fast 3D k-space sampling with high undersampling ratio. Combining the advantages of center-out spiral with the flexibility of the golden angle approach could have major implications for 3D real time imaging. 


\section{INTRODUCTION}

First introduced by Ahn \& al [1] and redefined by Meyer \& al [2], spiral readout has been used during the past decades in human and more recently in small animal imaging. Its use enables fast image acquisition with reduced motion and flow artifacts. Due to its high robustness, spiral sampling has been validated for applications such as functional MRI studies [3], coronary artery imaging [4] and most recently cardiac 3D T1 mapping on mice [5]. The ability to cover a large part of the kspace after one excitation allows faster acquisitions of images in two or three dimensions. Several methods combining spiral readouts with 3D imaging have been proposed. Among the existing techniques, the stack-of-spiral (SOS) approach allows fast space encoding by stacking spiral planes in the slice direction. This method has been applied to free breathing late gadolinium enhanced experiments $[6,7]$ and also to sodium imaging at high magnetic field [8]. The main advantages of SOS approach are the reduced scan times and the ability to acquire anisotropic field-of-view. However, SOS suffers from several drawbacks such as the slightly longer TE due to the spatial encoding in the third direction and the relative undersampling in the lower spatial frequencies when compared to 3D koosh-ball radial trajectories for example. Several spiral-encoding methods were developed to oversample the center of the k-space. First, twisted projection imaging (TPI) [9] enables fast signal acquisition with limited T2* effect. TPI has been used for sodium imaging in both cerebral tumors [10] and human articular cartilage [11]. Close to TPI, the cones method [12] allows fast kspace coverage using variable density trajectory. The cones approach was used in similar applications as TPI among which: sodium imaging of human patellar cartilage [13] and free-breathing 3D coronary artery imaging [14]. 
These space-encoding approach (spiral, TPI and cones) are compatible with an interleaved approach and may use a minimum time between the RF excitation and the signal acquisition, hence relevant for short and ultra short echo-time (UTE) imaging [15-17]. Although these techniques are very efficient for imaging and quantifying sodium concentration in various organs, the flexibility and robustness for undersampling has not been assessed yet. Among the existing methods, the goldenangle approach is particularly efficient to achieve pseudo-random sampling of the kspace [18] and was used in numerous applications. Moreover, its use allows significant reduction of eddy current artifacts [19] and is adapted for sliding window reconstruction. Few examples of combinations of golden-angle and 2D spiral readouts were proposed in the literature [20]. As far as we know, no sequence combining ultrashort echo-time and interleaved 3D spiral projected sampling with the golden angle approach was developed.

In this paper, we proposed to develop a k-space encoding method based on spiral projection imaging (SPI) $[21,22]$ allowing ultrashort echo-time (UTE) and interleaved acquisitions. This method was combined with an original sampling scheme using golden-angle method to pseudo-randomly sample the k-space. The aim of this study was (i) to demonstrate the feasibility to perform ultrashort echo-time imaging using projected spiral readout (ii) to show that the combination with interleaves and golden angle approach decrease the sensitivity to $\mathrm{T} 2 *$ decay and enable retrospective undersampling and (iii) to assess this new method in two different in vivo applications on mice at $7 \mathrm{~T}$. 


\section{METHODS}

Magnet, gradient system and RF coils

Experiments were performed on a 7-Tesla Bruker Biospec system (Ettlingen, Germany) equipped with a gradient system capable of $660 \mathrm{mT} / \mathrm{m}$ maximum strength and $110 \mu$ s rise time. A volume resonator $(86 \mathrm{~mm}$ inner diameter, active length 70 $\mathrm{mm})$ operating in quadrature mode was used for excitation, and a four-element $(2 \times 2)$ phased array surface coil (outer dimensions of one coil element: $12 \times 16 \mathrm{~mm}$, total outer dimensions: 26 × $21 \mathrm{~mm}$ ) was used for signal reception.

\section{K-space sampling and trajectory}

\section{Spiral design}

Spiral trajectories were designed as previously proposed by Cline \& al [23]. The trajectory was defined by $\boldsymbol{k}=\boldsymbol{A} \boldsymbol{\theta} \cdot \boldsymbol{e}^{(i \boldsymbol{\theta})}$ with A the number of spiral arms. As proposed by Cline \& al, the $\boldsymbol{\theta}(\boldsymbol{t})$ function was calculated by taking into account both the gradient amplitude (Eq [1]) and the rate limitations (Eq [2]).

$$
\begin{gathered}
\text { [1] } \theta_{S}(t)=\beta t^{2}\left(2+2\left(\frac{2 \beta}{3}\right)^{\frac{1}{6}} t^{\frac{1}{3}}+2\left(\frac{2 \beta}{3}\right)^{\frac{2}{3}} t^{\frac{4}{3}}\right)^{-1} \\
\text { [2] } \theta_{G}(t)=\sqrt{\varphi_{t}^{2}+2 \kappa\left(t-t_{\tau}\right)} \\
\text { with } \beta=\frac{\gamma G D}{M} \text { and } \kappa=\frac{\gamma S D}{M} .
\end{gathered}
$$

$\mathrm{G}$ was the maximum gradient intensity, $\mathrm{S}$ the maximum available slew-rate. $\varphi_{t}$ was the continuous rotation angle, $\gamma$ the gyromagnetic ratio, D the field-of-view (FOV), M the number of interleaves and $t_{\tau}$ the transition point between slew rate and gradient amplitude limited domains. 


\section{$3 D$ k-space sampling}

In order to generate a ball-like k-space, spiral gradients were applied along the three directions $(\mathrm{x}, \mathrm{y}, \mathrm{z})$. To minimize the $\mathrm{T} 2 *$ sensitivity induced by the long data collection, an interleave approach was developed to reduce the readout time and the number of sampled points per readout. Spiral interleaves were rotated about two axes for example $(\mathrm{x}, \mathrm{z})$. The global aim was to produce disk-like trajectories, where each disk contained spiral interleaves (rotated about $\mathrm{x}$ axis). The 3D encoding was performed by tilting the disks (rotation about $\mathrm{z}$ ) with a golden angle. Trajectories were calculated as:

$$
\begin{gathered}
\text { [3] } k_{x}=A \cdot \theta(t) \cdot \cos \left(\theta(t)+\phi_{x}(i)\right) \\
\text { [4] } k_{y}=A \cdot \theta(t) \cdot \sin \left(\theta(t)+\phi_{x}(i)\right) \cdot \cos \left(\phi_{z}(j)\right) \\
\text { [5] } k_{z}=A \cdot \theta(t) \cdot \sin \left(\theta(t)+\phi_{x}(i)\right) \cdot \sin \left(\phi_{z}(j)\right) \\
\phi_{x}(i)=\frac{2 \pi}{N_{\text {Interleave }}} \cdot i \text { and } \phi_{z}(j)=\pi \cdot(3-\sqrt{5}) \cdot j
\end{gathered}
$$

The $\theta$ function was chosen as previously explained. $i$ was a variable corresponding to the number of interleaves per disks (from 1 to $N_{\text {interleave }}$ ). $j$ was a variable which corresponded to the number of disks (from 1 to $N_{\text {disks }}$ ). Fig.1 shows an example of the k-space filling order for 10 disks of 5 interleaves. The first acquired spiral corresponded to the first TR number and was associated with a rotation angle around $\mathrm{z}$ axis $\left(\phi_{z}(1)\right)$ and $\mathrm{x}$ axis $\left(\phi_{x}(1)\right)$. For the following TRs, each spiral was associated with a different couple of $\phi_{z}$ and $\phi_{x}$ which were calculated as explained in Fig.1. Fig.2 shows a 3D view of k-space filling. The first acquired spiral (TR number 1) corresponded to the first interleave ( $\left.\mathrm{I}_{1}\right)$ of the first disk ( $\left.\mathrm{D}_{1}\right)$. Then, the second spiral (TR number 2) was associated with a second couple of angles $\left(\phi_{z}(2)\right.$ and $\left.\phi_{x}(2)\right)$ and 
corresponded to the second interleave ( $\left.\mathrm{I}_{2}\right)$ of the second disk $\left(\mathrm{D}_{2}\right)$. The use of a golden-angle $\left(\phi_{z}(j)\right)$ allowed to produce a ball-like shaped k-space in a short acquisition time (from TR number 1 to 10 ). Then, at TR number 11, the second interleave of the first disk was acquired. This acquisition scheme was repeated until the filling of the k-space made of 10 disks of 5 interleaves, as illustrated in Fig.3. For each experiment, the k-space was acquired with a fixed number of 128 disks ( $\left.\mathrm{N}_{\text {disks }}\right)$ and a variable couple of number of interleaves and number of data points per interleave (Ninterleaves $\left./ \mathrm{N}_{\text {points}}\right)$.

\section{Reconstruction process}

K-space data were regridded with an oversampling of 2 using a Kaiser-Bessel kernel [24]. Final images were reconstructed by applying a Fast Fourier Transform (FFT). Each phased array receiver magnitude image was reconstructed using the method described above and then combined with a sum of squares reconstruction. The reconstruction algorithm was implemented in MATLAB (MathWorks, Natick, MA, USA). Prior to regridding, spiral trajectories were measured and averaged on a phantom filled with water to minimize lack of SNR, as presented by Beaumont \& al [25]. Measured trajectories were used during the gridding process allowing to correct both eddy currents and the gradient delay errors.

Simulations and image analysis Point spread function (PSF)

As previously mentioned, the long data collection time induced by the spiral readouts generates a high sensitivity to $\mathrm{T} 2 *$ decay which may alter the spatial resolution. To quantify the effect of $\mathrm{T} 2 *$ decay on the spatial resolution of our images, 
the PSF was calculated using $\mathrm{N}_{\text {disks }}=128$, with variable $\mathrm{N}_{\text {interleaves }} / \mathrm{N}_{\text {points }}=128 / 480$, 64/492, 32/607, 16/1054. The PSF was calculated by performing gridding on a simulated k-space matrix with ones along the spiral trajectory followed by a 3D-FFT as proposed by Feng \& al [26]. Then, simulated data were multiplied by the exponential T2* decay $\left(\exp ^{\left(\frac{-t}{T 2 *}\right)}\right)$ by setting different $\mathrm{T} 2 *$ values $(0.1,0.3,0.5,1$, 2.5, and $5 \mathrm{~ms}$ ) and the PSF full-width at half maximum (FWHM) was measured.

\section{Quantitative Image Sharpness Analysis}

Three phantoms with increased concentrations of Ultrasmall superparamagnetic Iron Oxide (USPIO) $(1,3,5 \mathrm{mM})$ were suspended in agarose gel $(1 \%)$. Then image acquisitions were performed using the following parameters: TR/TE: 5/0.025 ms, imaging pulse: block pulse $0.05 \mathrm{~ms}$, Flip-Angle $(\mathrm{FA})=10^{\circ}$, matrix: $128 \times 128 \times 128$, field-of-view: $20 \times 20$ × $20 \mathrm{~mm} 3$, spatial resolution: 152 × 152 × $152 \mu \mathrm{m} 3$, bandwidth/pixel: $2344 \mathrm{~Hz} /$ pixel, $\mathrm{N}_{\text {disks }}=128, \mathrm{~N}_{\text {interleaves }} / \mathrm{N}_{\text {points }}=128 / 480,64 / 492$, 32/607, 16/1054. After image acquisition, each image was reconstructed using random undersampling with increasing factors $(2,4,6,8$ and 10). For example, an undersampling factor of 8 corresponded to an image reconstructed with $12,5 \%$ of the available raw datas. For each reconstructed image, using MATLAB software, ten intensity profiles were measured across the gel and the inserted plastic material (lego ${ }^{\circledR}$, Billund, Denmark) at ten different locations. The maximum and minimum intensity values across the profile boundary were determined (Imax and Imin), which corresponded to gel and plastic pixel intensities, respectively. Next, the distance, d, between $0.8\left(I_{\max }-I_{\min }\right)+I_{\min }$ and $0.2\left(I_{\max }-I_{\min }\right)+I_{\min }$ across the profile was measured and the sharpness value for that particular profile was reported as $(1 / \mathrm{d}) \mathrm{mm}-1$. The sharpness corresponding to each image was the average of the sharpnesses calculated 
from the ten line profiles. This quantification strategy was similar to that used by Shea \& al [27] and Larson \& al [28].

\section{In-vivo experiments}

\section{Brain imaging}

In order to estimate the in vivo robustness of our method, images were acquired on the brain of five healthy mice $(\mathrm{C} 57 \mathrm{Bl} / 6$, Charles River, Paris, France). The mice respiration was followed using a balloon (SA Instruments, Stony Brook, NY, USA) fixed on the abdomen. In order to visualize the time-of-flight effect, a slice selection was added prior to signal acquisition leading to an increase in TE. The following acquisition parameters were used, TR/TE: 5/1.3 ms, imaging pulse: Cardinal sine $1 \mathrm{~ms}, \mathrm{FA}=10^{\circ}$, matrix: $128 \times 128 \times 128$, field-of-view: $20 \times 20 \times 20 \mathrm{~mm}$, spatial resolution: $152 \times 152 \times 152 \mu \mathrm{m} 3$, bandwidth/pixel: $2344 \mathrm{~Hz}, \mathrm{~N}_{\text {disks }}=128$, number of interleaves per planes: Ninterleaves $/ \mathrm{N}_{\text {points }}=128 / 480,64 / 492,32 / 607,16 / 1054$.

\section{Self-gated liver imaging}

To apply our method on moving organs, images were acquired in the abdomen of five healthy mice (C57B1/6, Charles River, Paris, France). Respiration was monitored as previously explained. To detect signal fluctuations induced by breathing, the Analog to Digital Converter (ADC) was turned on immediately after the RF pulse. By extracting the first data point from the FID and plotting its intensity as a function of acquisition time, a curve was obtained and respiration phases were identified. Raw data were re-ordered as a function of their position in the breathing cycle allowing to reconstruct cine frames free of motion artifact. For these experiments, mice were injected with $100 \mu \mathrm{L}$ of Sinerem (Guerbet, Aulnay-sous-bois, 
France) at $200 \mu$ mol.kg-1. The following acquisition parameters were used, TR/TE: 5/0.025 ms, imaging pulse: Block pulse $0.05 \mathrm{~ms}, \mathrm{FA}=25^{\circ}$, matrix: $128 \times 128 \times 128$, field-of-view: 20 × 20 × $20 \mathrm{~mm} 2$, spatial resolution: 152 × 152 × $152 \mu \mathrm{m} 3$, bandwidth/pixel: $2344 \mathrm{~Hz}, \mathrm{~N}_{\text {disks }}=128, \mathrm{~N}_{\text {interleaves} /} / \mathrm{N}_{\text {points }}=128 / 492$. Number of repetition: 10 . 


\section{RESULTS}

PSF simulation and $T 2 *$ effect

The full-width at half maximum (FWHM) of the PSF are shown in Table.1 for different $\mathrm{N}_{\text {interleaves }} / \mathrm{N}_{\text {points }}$ and increasing T2*. As expected, FWHM decreased with shorter T2* values. For T2* shorter than $1 \mathrm{~ms}$, the PSF was narrower when a higher number of interleaves was used. For example, FWHM increased from 6.8 with $N_{\text {interleaves }}=128$ to 12.1 with $\mathrm{Ninterleaves}=16$ when $\mathrm{T} 2 *$ was $0.1 \mathrm{~ms}$. Interestingly, for longer T2*, no significant difference was observed. Indeed, for T2* of 5ms, FWHM was 1.6 with Ninterleaves $=128$ versus 1.5 with Ninterleaves $=16$.

\section{Sharpness and random undersampling}

Representative images using Ninterleaves $/ \mathrm{N}_{\text {points }}=128 / 492$ are shown in Fig.4. Images were acquired on phantoms containing increasing USPIO concentrations $(1,3$ and $5 \mathrm{mM}$ ). Without undersampling, increasing USPIO concentrations led to blurring artifacts (arrow). Moreover, whatever the USPIO concentration, the blurring effect increased with the undersampling factor. At the higher undersampling ratio, streaking artifacts appeared on the magnitude images (dashed arrow). For all the images acquired with the differents $\mathrm{Ninterleaves} / \mathrm{N}_{\text {points, }}$ the sharpness was calculated. Every sharpness was normalized to the maximum and represented on Fig.5. Whatever the number of interleaves per disk, the sharpness expectedly decreased by increasing the undersampling factor. The sharpness attenuation was similar for all different Ninterleaves $/ \mathrm{N}_{\text {points }}$ whatever the USPIO concentration. For example, at the higher undersampling factor and the $5 \mathrm{mM}$ concentration of USPIO, the normalized sharpness was 0.4 with Ninterleaves $=128,0.4$ with $\mathrm{N}_{\text {interleaves }}=64,0.4$ with Ninterleaves $=32$ and 0.48 with 
Ninterleaves $=16$. It should be noted that the sharpness values were intrinsically lower by decreasing the number of interleave per disk (data not shown).

\section{Brain imaging}

Images were acquired on healthy mice with variable $\mathrm{N}_{\text {interleaves }} / \mathrm{N}_{\text {points. The }}$ resulting images are shown in Fig.6. Using Ninterleaves=128 and 64, the Time of Flight effect and the spatial resolution were high enough to distinguish the middle cerebral artery with branches, as shown by the arrows in Fig.6.a. For lower number of interleaves, the middle cerebral artery could not be visualized demonstrating the loss of spatial resolution certainly due to off-resonance sensitivity. However, larger vessels, such as carotid arteries could be detected whatever the number of interleaves per disk, as shown by the dashed arrow in Fig.6.b. The total scan times to acquire these $3 \mathrm{D}$ images were $1 \mathrm{~min} 20 \mathrm{~s}, 40 \mathrm{~s}, 20 \mathrm{~s}$ and $10 \mathrm{~s}$ corresponding to $\mathrm{N}_{\text {interleaves }} / \mathrm{N}_{\text {points }}=$ $128 / 480,64 / 492,32 / 607,16 / 1054$, respectively.

\section{Respiratory self-gated 3D liver angiography}

Images were acquired on healthy awake mice breathing at 120 inspirations per minute. Twenty frames were reconstructed per respiratory cycle. As shown in Fig.7, the injection of USPIO coupled with the ultrashort-echo time of our sequence generated a positive contrast between the blood vessels and the liver. Moreover, after the self-gated reconstruction, the whole liver angiography was detected (arrows) without any blurring effect caused by breathing. The overall acquisition time was 12 minutes. The mean number of projections per k-space was 7000 corresponding to an undersampling factor of 2 compared to the non-gated reference $k$-space of $\mathrm{N}_{\text {disk }}=128$, Ninterleaves $/ \mathrm{N}_{\text {points }}=128 / 492$. The movie corresponding to the images shown in Fig.7 
were added in the supplementary material. (SuppMat.1 : axial view ; SuppMat.2 : sagittal view).

\section{DISCUSSION}

In this project, an original MRI sequence based on hybrid spiral/radial also called spiral projected imaging has been developed. This method allowed ultra-short echo time (UTE) and both prospective and retrospective undersampling due to the use of the golden-angle approach. First, the sensitivity to $\mathrm{T} 2 *$ and the impact on point spread function were evaluated. The robustness for undersampling was quantified by measuring the sharpness on images reconstructed with reduced numbers of spiral projections. Then, this method was assayed in vivo in mice for: whole brain angiography and respiratory self gated 3D liver angiography at 7T.

The major advantage of combining spiral and radial acquisitions versus conventional stack-of-spiral approach was the k-space center oversampling. Indeed, each spiral was registered from the center to the peripheral parts of the k-space allowing to decrease motion sensitivity while increasing signal-to-noise ratio. Moreover, such an encoding scheme did not require the use of an additional cartesian encoding in the third direction allowing maximal TE reduction. This lead to a decrease in sensitivity to blood flow particularly strong for small animal angiography. Few methods closed to spiral projected imaging (SPI), namely cones and twisted projections, allow to oversample the center of the $\mathrm{k}$-space. These methods have been previously used to quantify short $\mathrm{T} 2 *$ nucleus, such as sodium, in both brain and cartilage $[13,16]$. However, none of these techniques were applied in the abdomen which is an area strongly affected by motion. In this paper, after demonstrating that even at high concentration of USPIO, no distortion or severe blurring artifact affected 
in vitro images. The interest of combining UTE, USPIO injection and spiral projected imaging was demonstrated for 3D respiratory-self gated liver angiography. As shown in Fig.7, blood vessels were visualized with a positive contrast without flow artifact. All these results showed the interest of using this method for abdominal imaging which is a major challenge in small animals. Potential applications on lung and osteoarticular imaging should also be considered.

Coupling SPI protocol with the golden-angle approach also allowed to perform both prospective and retrospective undersampling. Indeed, this encoding strategy allowed undersampling in both radial (by removing spiral interleaves in a given disk) and azimuthal (by recording less disks) directions. The robustness to undersampling was first demonstrated in vitro. Furthermore, even with an undersampling factor of 10 (meaning that $90 \%$ of raw data were removed for image reconstruction), the sharpness only decreased by a factor of two. This result was validated in vivo with a self-gated reconstruction of a 3D mouse liver angiography which was performed using a random undersampling (in both radial and azimuthal direction). This intrinsic robustness to undersampling makes it possible to consider dynamic applications such as first-pass perfusion imaging as well as motion correction for abdominal investigations. Further experiments are currently in progress to investigate the ability to combine sliding window reconstruction with the SPI method developed in this study. Moreover, several techniques were recently proposed in the literature for combining twisted projection [29] and spiral [30] with both parallel imaging and compressed sensing algorithm. Coupling SPI with powerful reconstruction procedure should allow very fast data collection and image reconstruction.

Although this method showed promising results in vitro and in vivo several issues still remain. First, the long readout time generated by spiral acquisition is 
double-edged especially at high magnetic field. Certainly, it allows fast data collection but the resulting images may suffer from severe blurring artifact. To overcome this limitation, a combination of high bandwidth $(300 \mathrm{kHz})$ and an interleaved approach was proposed. However, it should be noted that longer T2* observed at lower magnetic field $(1,5 \mathrm{~T})$ may lead to faster signal acquisition by significantly reducing the number of interleaves per disk. The second limitation related to the use of spiral readout was the sensitivity to off-resonance and chemical shift artifacts which should be taken care of for further investigations in the abdomen. Several methods have already been developed to suppress fat signal contribution on both clinical [31] and pre-clinical scanners [32] and may be easily combined with the sampling strategy proposed in this project. The third drawback of SPI method was related to the trajectories themselves. Indeed, to obtain a ball-like k-space, the gradients were activated in the three directions $(\mathrm{x}, \mathrm{y}, \mathrm{z})$. This may lead to trajectory errors due to eddy-currents and gradient delay approximations. To correct such errors, trajectories were measured prior to signal acquisition and used during the regridding procedure. Finally, even though isotropic FOV required for signal acquisition may be an advantage for whole body applications it can be a limiting factor for imaging restricted body areas. However, a previous developed method should be applied on this sampling strategy to acquire anisotropic FOV [33].

The acquisition protocol proposed in this project displayed interesting features especially in terms of acquisition time, robustness for undersampling and flexibility due to spiral gradients. It should be noted that spiral readout can be either in or out allowing variations of the echo-time values leading to potential applications for fMRI studies. Recent works demonstrated the interest of using fast spiral acquisitions for such applications $[34,35]$. But, as far as we know, no spiral projected imaging for 
fMRI has yet been reported in the literature. Moreover, the ability to switch from very short to very long echo times presents a great advantage for measuring in vivo temperature using phase image differences. The use of 2D spiral acquisition for this kind of application has already been discussed by Stafford \& al [36]. However, it should be very interesting to monitor temperature variations in $3 \mathrm{D}$ and we believe that the proposed method provides enough flexibility and robustness to properly retrieve this information. Finally, the fast 3D k-space sampling enabled by this acquisition strategy may have implications in $3 \mathrm{D}$ real-time imaging. The usefulness of spiral trajectory has already been demonstrated for 2D imaging in applications such as real time bSSFP heart imaging [37] and for distortion correction [38]. However, no method allowing $3 \mathrm{D}$ real time imaging has been proposed in the literature yet. This could have major implications especially on applications such as catheter tracking [39].

\section{CONCLUSION}

In this study, an innovative method based on spiral projection imaging coupled with a golden-angle approach and ultrashort echo-time has been proposed. The robustness for undersampling and the sensitivity to short $\mathrm{T} 2 *$ was evaluated on both in vitro and in vivo experiments. This protocol was checked for 3D angiography on both mouse brain and liver. The ultrashort echo-time coupled with the high robustness for undersampling provides major assets to this sequence to be used for a wide range of application where flexibility and short acquisition time are needed. 


\section{ACKNOWLEDGMENTS}

This work was supported by a public grant, Translational Research and Advanced Imaging Laboratory, which is part of the French National Research Agency's Investments for the Future Program ("NewFISP”; ANR- 10-LABX-57). 


\section{REFERENCES}

[1] Ahn CB, Kim JH, Cho ZH. High-speed spiral-scan echo planar NMR imaging-I. IEEE Trans Med Imaging 1986;5:2-7.

[2] Meyer $\mathrm{CH}, \mathrm{Hu}$ BS, Nishimura DG, Macovski A. Fast spiral coronary artery imaging. Magn Reson Med 1992;28:202-213.

[3] Glover GH. Spiral imaging in fMRI. Neuroimage 2012;62:706-712.

[4] Yang PC, Meyer CH, Terashima M, Kaji S, McConnell MV, Macovski A, Pauly JM, Nishimura DG, Hu BS. Spiral magnetic resonance coronary angiography with rapid real-time localization. J Am Coll Cardiol 2003;41:1134-1141.

[5] Castets CR, Ribot EJ, Lefrançois W, Trotier AJ, Thiaudière E, Franconi JM,

Miraux S. Fast and robust 3D T1 mapping using spiral encoding and steady RF excitation at $7 \mathrm{~T}$ : application to cardiac manganese enhanced MRI (MEMRI) in mice. NMR Biomed 2015;28:881-889.

[6] Shin, Taehoon, Michael Lustig, Dwight G. Nishimura, and Bob S. Hu. Rapid Single-Breath-Hold 3D Late Gadolinium Enhancement Cardiac MRI Using a Stackof-Spirals Acquisition. J Magn Reson Imaging 2014;40:1496-1502.

[7] Pierce, Iain T., Jennifer Keegan, Peter Drivas, Peter D. Gatehouse, and David N. Firmin. "Free-Breathing 3D Late Gadolinium Enhancement Imaging of the Left 
Ventricle Using a Stack of Spirals at 3T.” J Magn Reson Imaging 2015;41:10301037.

[8] Qian, Yongxian, Tiejun Zhao, Hai Zheng, Jonathan Weimer, and Fernando E. Boada. High-Resolution Sodium Imaging of Human Brain at 7 T. Magn Reson Med 2012;68:227-233.

[9] Boada, Fernando E., Gary X. Shen, Sam Y. Chang, and Keith R. Thulborn. Spectrally Weighted Twisted Projection Imaging: Reducing T2 Signal Attenuation Effects in Fast Three-Dimensional Sodium Imaging. Magn Reson Med 1997;38:1022-1028.

[10] Thulborn KR, Davis D, Adams H, Gindin T, Zhou J. Quantitative tissue sodium concentration mapping of the growth of focal cerebral tumors with sodium magnetic resonance imaging. Magn Reson Med 1999;41:351-359.

[11] Borthakur A, Hancu I, Boada FE, Shen GX, Shapiro EM, Reddy R. In vivo triple quantum filtered twisted projection sodium MRI of human articular cartilage. J Magn Reson 1999;141:286-290.

[12] Boada F, Gillen J, Shen G, Chang S, Thulborn K. Fast three dimensional sodium imaging. Magn Reson Med 1997;37:706 -715.

[13] Staroswiecki, Ernesto, Neal K. Bangerter, Paul T. Gurney, Thomas Grafendorfer, Garry E. Gold, and Brian A. Hargreaves. In Vivo Sodium Imaging of Human Patellar 
Cartilage with a 3D Cones Sequence at $3 \mathrm{~T}$ and $7 \mathrm{~T}$. J Magn Reson Imaging 2010;32:446-451.

[14] Wu, Holden H., Paul T. Gurney, Bob S. Hu, Dwight G. Nishimura, and Michael V. McConnell. Free-Breathing Multiphase Whole-Heart Coronary MR Angiography Using Image-Based Navigators and Three-Dimensional Cones Imaging. Magn Reson Med 2013;69:1083-1093.

[15] Qian, Yongxian, and Fernando E. Boada. Acquisition-Weighted Stack of Spirals for Fast High-Resolution Three-Dimensional Ultra-Short Echo Time MR Imaging. Magn Reson Med 2008;60:135-145.

[16] Riemer, Frank, BhavanaS. Solanky, Christian Stehning, Matthew Clemence, ClaudiaA.M. Wheeler-Kingshott, and Xavier Golay. Sodium (23Na) Ultra-Short Echo Time Imaging in the Human Brain Using a 3D-Cones Trajectory. MAGMA $2014 ; 27: 35-46$.

[17] Konstandin S, Krämer P, Günther M, Schad LR. Sodium magnetic resonance imaging using ultra-short echo time sequences with anisotropic resolution and uniform k-space sampling. Magn Reson Imaging 2015;33:319-327.

[18] Winkelmann S, Schaeffter T, Koehler T, Eggers H, Doessel O. An optimal radial profile order based on the Golden Ratio for time-resolved MRI. IEEE Trans Med Imaging 2007;26:68-76. 
[19] Wundrak S, Paul J, Ulrici J, Hell E, Rasche V. A Small Surrogate for the Golden Angle in Time-Resolved Radial MRI Based on Generalized Fibonacci Sequences. IEEE Trans Med Imaging 2015;34:1262-1269.

[20] Turley DC, Pipe JG. Distributed spirals: a new class of three-dimensional kspace trajectories. Magn Reson Med 2013;70:413-419.

[21] Irarrazabal P, Nishimura DG. Fast three dimensional magnetic resonance imaging. Magn Reson Med 1995;33:656-662.

[22] Pipe JG, Koladia KV. Spiral Projection Imaging: a new fast 3D trajectory. In: Proceedings of the 13th International Society of Magnetic Resonance in Medicine Meeting, Miami Beach, FL, USA, 2005; Abstract 2402.

[23] Cline HE, Zong X, Gai N. Design of a logarithmic k-space spiral trajectory. Magn Reson Med 2001;46:1130-1135.

[24] Beatty PJ, Nishimura DG, Pauly JM. Rapid gridding reconstruction with a minimal oversampling ratio. IEEE Trans Med Imaging 2005;24:799-808.

[25] Beaumont M, Lamalle L, Segebarth C, Barbier EL.Improved k-space trajectory measurement with signal shifting. Magn Reson Med 2007;58:200-205.

[26] Feng L, Grimm R, Block KT, Chandarana H, Kim S, Xu J, Axel L, Sodickson DK, Otazo R. Golden-angle radial sparse parallel MRI: combination of compressed 
sensing, parallel imaging, and golden-angle radial sampling for fast and flexible dynamic volumetric MRI. Magn Reson Med 2014; 72:707-717.

[27] Shea SM, Kroeker RM, Deshpande V, Laub G, Zheng J, Finn JP, Li D. Coronary artery imaging: 3D segmented k-space data acquisition with multiple breath-holds and real-time slab following. J Magn Reson Imaging 2001;13:301-307.

[28] Larson AC, Kellman P, Arai A, Hirsch GA, McVeigh E, Li D, Simonetti OP. Preliminary investigation of respiratory self-gating for free-breathing segmented cine MRI. Magn Reson Med 2005;53:159-168.

[29] Addy NO, Ingle RR, Wu HH, Hu BS, Nishimura DG. High-resolution variabledensity 3D cones coronary MRA. Magn Reson Med 2015;74: 614-621.

[30] Dyvorne H, Knight-Greenfield A, Jajamovich G, Besa C, Cui Y, Stalder A, Markl M, Taouli B. Abdominal 4D flow MR imaging in a breath hold: combination of spiral sampling and dynamic compressed sensing for highly accelerated acquisition. Radiology 2015;275:245-254.

[31] Börnert P, Koken P, Eggers H. Spiral water-fat imaging with integrated offresonance correction on a clinical scanner. J Magn Reson Imaging 2010;32:12621267.

[32] Ragan DK, Bankson JA. Two-point Dixon technique provides robust fat suppression for multi-mouse imaging. J Magn Reson Imaging 2010;31:510-514. 
[33] Larson PZ, Gurney PT, Nishimura DG. Anisotropic field-of-views in radial imaging. IEEE Trans Med Imaging 2008;27:47-57.

[34] Glover GH. Spiral imaging in fMRI. Neuroimage 2012;62:706-712.

[35] Jung Y, Samsonov AA, Liu TT, Buracas GT. High efficiency multishot interleaved spiral-in/out: acquisition for high-resolution BOLD fMRI. Magn Reson Med 2013;70:420-428.

[36] Stafford RJ, Hazle JD, Glover GH. Monitoring of high-intensity focused ultrasound-induced temperature changes in vitro using an interleaved spiral acquisition. Magn Reson Med 2000;43:909-912.

[37] Feng X, Salerno M, Kramer CM, Meyer CH. Non-Cartesian balanced steadystate free precession pulse sequences for real-time cardiac MRI. Magn Reson Med 2015. doi:10.1002/mrm.25738.

[38] Campbell-Washburn AE, Xue H, Lederman RJ, Faranesh AZ, Hansen MS. Realtime distortion correction of spiral and echo planar images using the gradient system impulse response function. Magn Reson Med 2015. doi: 10.1002/mrm.25788.

[39] Whiting N, Hu J, Shah JV, Cassidy MC, Cressman E, Zacharias Millward N, Menter DG, Marcus CM, Bhattacharya PK. Real-Time MRI-Guided Catheter Tracking Using Hyperpolarized Silicon Particles. Sci Rep 2015;5:12842. 



\section{FIGURE CAPTIONS}

Figure 1. Acquisition method to fill a 3D k-space using 10 disks with 5 interleaves. The colored numbers represent the TR number. The first acquired spiral (at TR number 1) was associated with a couple of rotation angles around $\mathrm{z}$ axis $\left(\phi_{z}(1)\right)$ and $\mathrm{x}$ axis $\left(\phi_{x}(1)\right)$. Then, each of the following recorded spirals were associated with a different couple of rotation angles $\phi_{z}(j)$ and $\phi_{x}(i)$. Colored number matches with the spiral colors in Fig.2.

Figure 2. Example of 3D k-space filling for ten disks of five interleaves per disk. At the first TR, the first interleave ( $\left.\mathrm{I}_{1}\right)$ of the first disk (D1) was recorded. Then, the next acquired spiral was rotated along $\mathrm{x}$ axis $\left(\phi_{x}(2)\right)$ and $\mathrm{z}$ axis $\left(\phi_{z}(2)\right)$ as synthetized by the arrows and corresponded to the second interleave $\left(\mathrm{I}_{2}\right)$ of the second disk $\left(\mathrm{D}_{2}\right)$. This acquisition was repeated to obtain a ball-like complete k-space composed by 10 disks, each one made of 5 interleaves (bottom of the figure).

Figure 3. In-plane view of the 10 disks composed of 5 coplanar interleaves. acquired at different TR numbers corresponding to the columns of Fig.1.

Figure 4. Images of phantoms acquired with $\mathrm{N}_{\text {disks }}=128$ and $\mathrm{N}_{\text {interleaves }} / \mathrm{N}_{\text {points }}=$ 128/480. Three different concentrations of USPIO $(1,3,5 \mathrm{mM})$ were used. Images were reconstructed by randomly undersampling $\mathrm{k}$-space before reconstruction (undersampling factor: 2, 4, 6, 8, 10). Dashed arrow points at streaking artifact, white arrow point a blurring effect induced by very short $\mathrm{T} 2 *$. 
Figure 5. Normalized sharpness in function of undersampling factor for increasing concentrations of USPIO $(1,3,5 \mathrm{mM})$ with variable number of interleaves per disk a) Ninterleaves $/ \mathrm{N}_{\text {points }}=128 / 480$, b) Ninterleaves $/ \mathrm{N}_{\text {points }}=64 / 492$, c) $\mathrm{N}_{\text {interleaves }} / \mathrm{N}_{\text {points }}=$ $32 / 607$ d) Ninterleaves $/ N_{\text {points }}=16 / 1054$. The sharpnesses were measured on the phantom showed in Fig.4. Scale bar represents $1 \mathrm{~cm}$.

Figure 6. Sagittal view of images acquired on a healthy mouse with variable Ninterleaves $/ \mathrm{N}_{\text {points }}=128 / 480,64 / 492,32 / 607,16 / 1054$. The rows stand for two different slices. a) white arrows point at cerebral middle artery, b) dashed arrow point at carotid artery. Scale bar represents $1 \mathrm{~cm}$.

Figure 7. Self-gated 3D liver angiography (axial and sagittal views). Each extracted image represents a cine frame synchronized with breathing. White arrows point at regions of interest which move during the breathing cycle (inspiration and expiration). The corresponding films were added in the supplementary material part. Scale bar represents $1 \mathrm{~cm}$.

SuppMat.1 Movie generated from the twenty cine images of the self-gated 3D liver angiography (axial view).

SuppMat.2 Movie generated from the twenty cine images of the self-gated 3D liver angiography (sagittal view). 


\begin{tabular}{ccccc} 
& \multicolumn{5}{c}{ FWHM of Point Spread Function (PSF) } \\
\cline { 2 - 5 } $\mathrm{T} 2^{\star} / \mathrm{ms}$ & $128 \mathrm{int} /$ disk & 64 int / disk & 32 int / disk & 16 int / disk \\
\hline 0.1 & 6.8 & 7.1 & 7.6 & 12.1 \\
0.3 & 3.0 & 3.1 & 3.4 & 5.3 \\
0.5 & 2.4 & 2.5 & 2.6 & 3.6 \\
1 & 1.8 & 1.9 & 1.9 & 2.4 \\
2.5 & 1.7 & 1.7 & 1.5 & 1.6 \\
5 & 1.6 & 1.6 & 1.4 & 1.5 \\
\hline
\end{tabular}

Table.1 Full width at half maximum of the Point Spread Function (PSF) with variable number of interleaves per disk $(128,64,32,16)$ and increasing $\mathrm{T} 2 *(0.1,0.3,0.5,1,2.5,5$ $\mathrm{ms})$. 


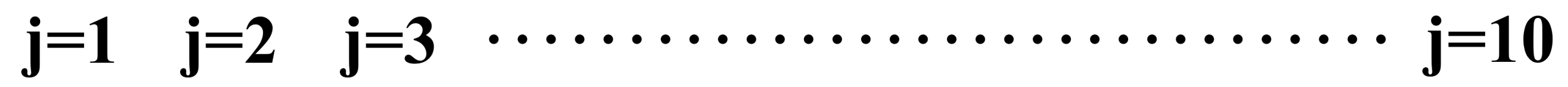

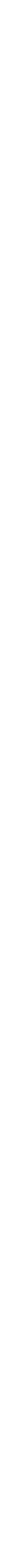




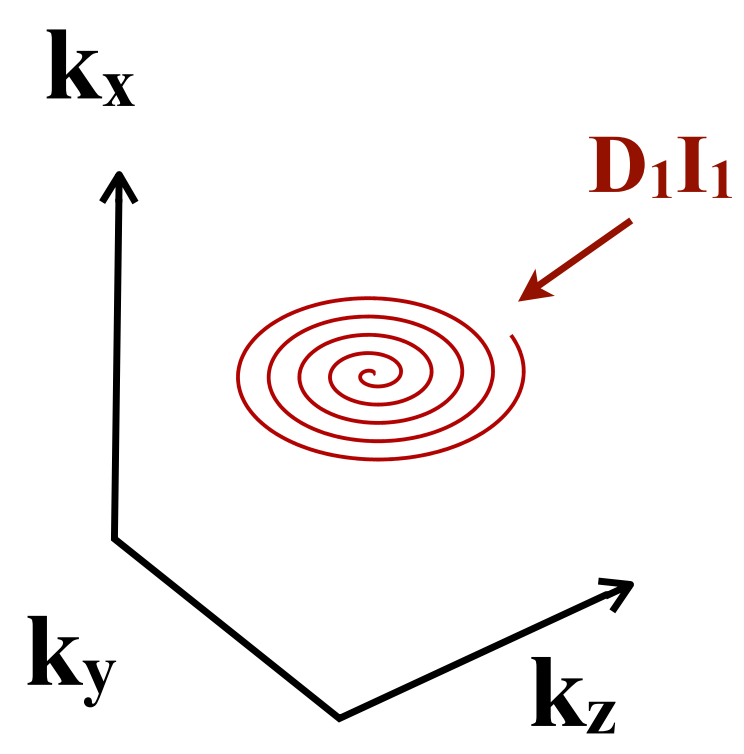

TR number $=1$

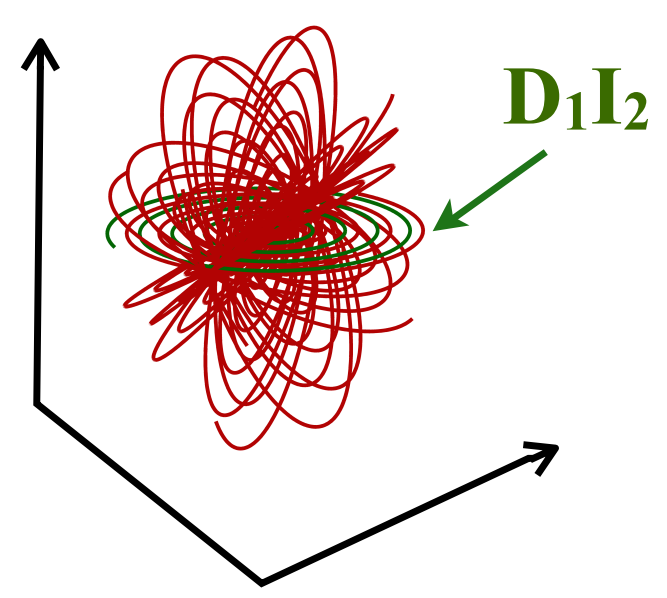

TR number 1, 2, 3...11

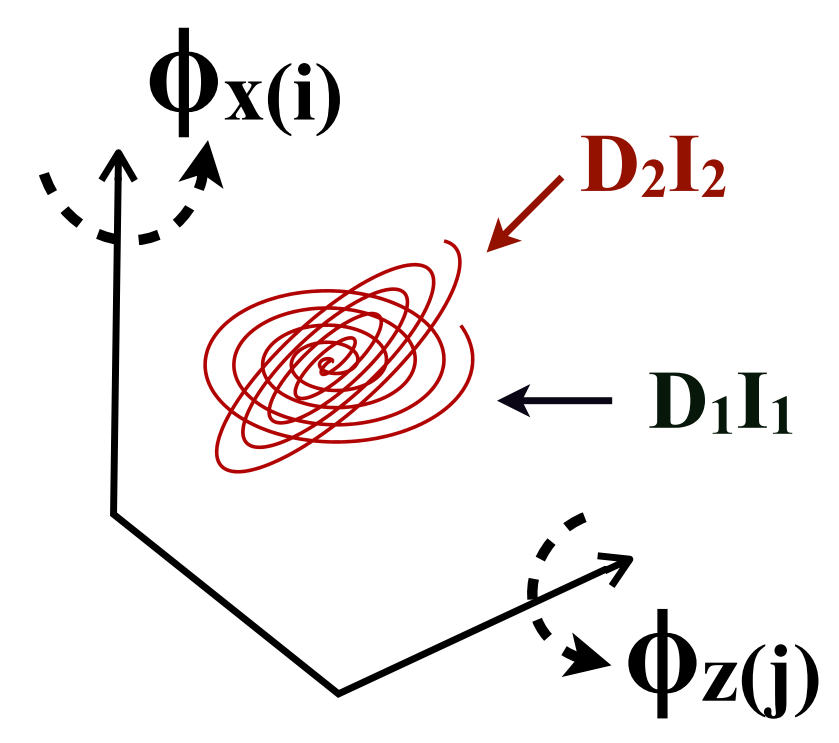

TR number 1, 2

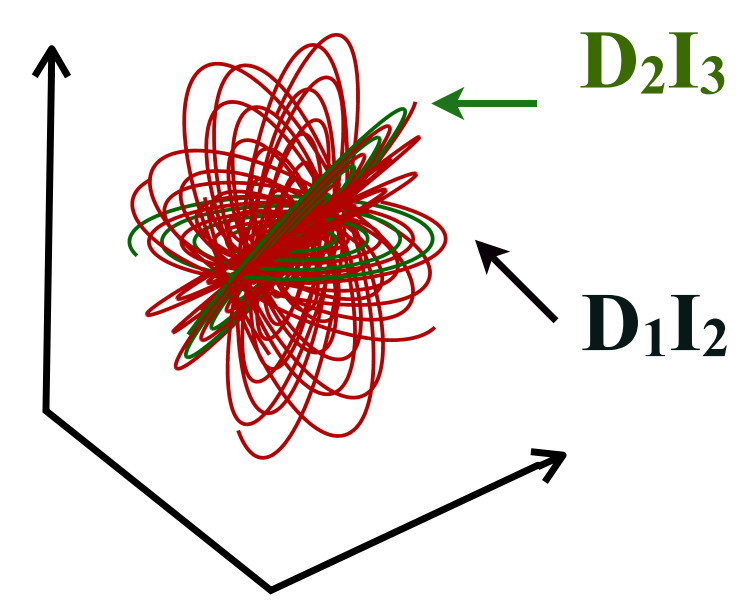

TR number 1, 2, 3...12

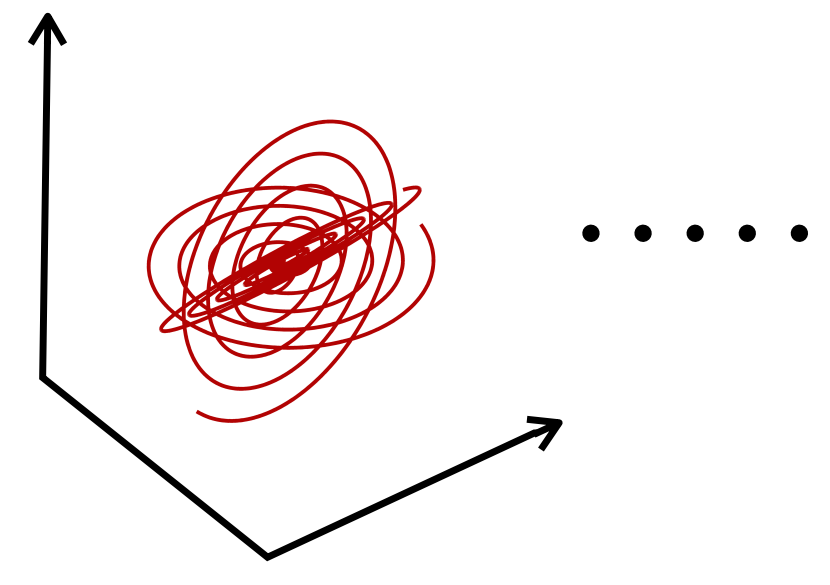

TR number 1, 2, 3

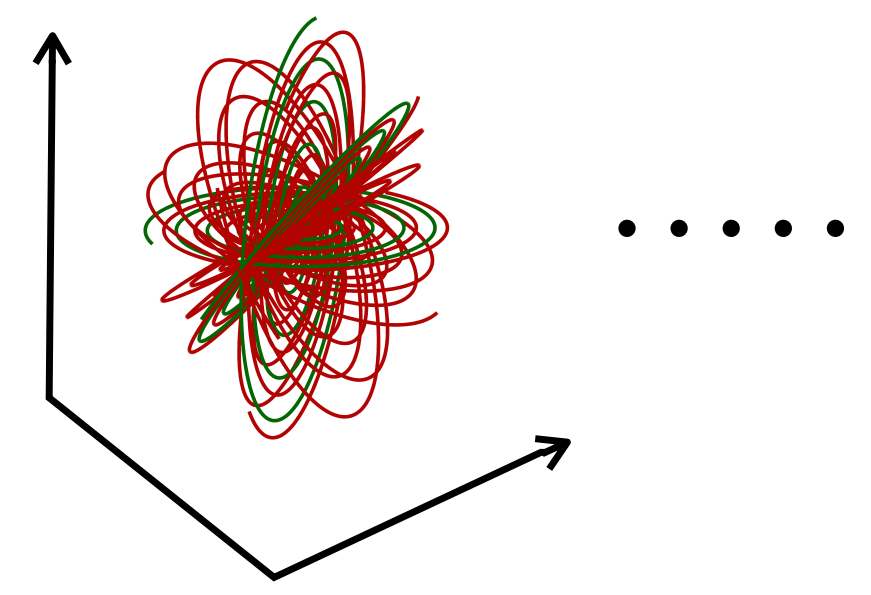

TR number 1, 2, 3...13

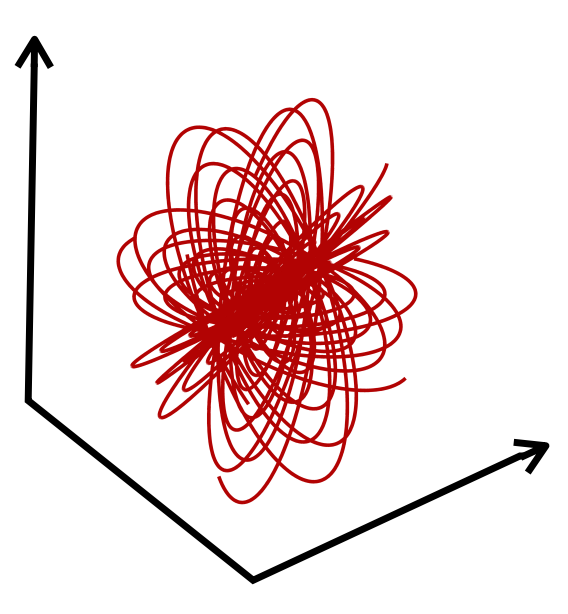

TR number 1, 2, 3...10

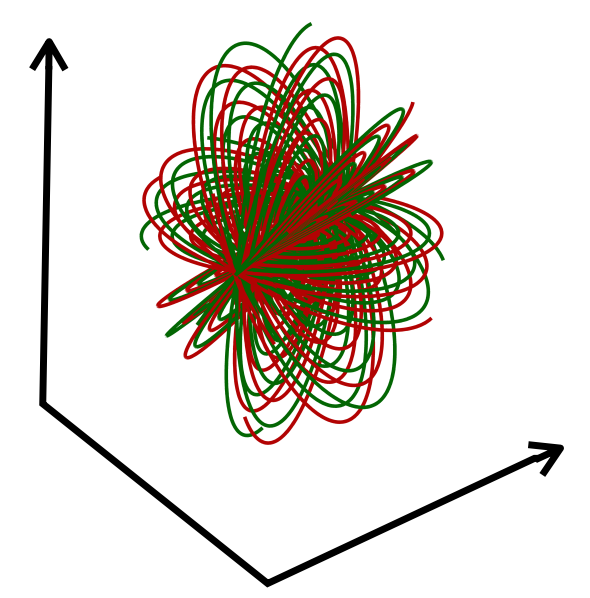

TR number 1, 2, 3...20
$D_{j}=$ Disk number ' $\mathbf{j}$ ' $I_{i}=$ Interleave number ' $i$ ' complete k-space $=$

10 Disks with 5 Interleaves

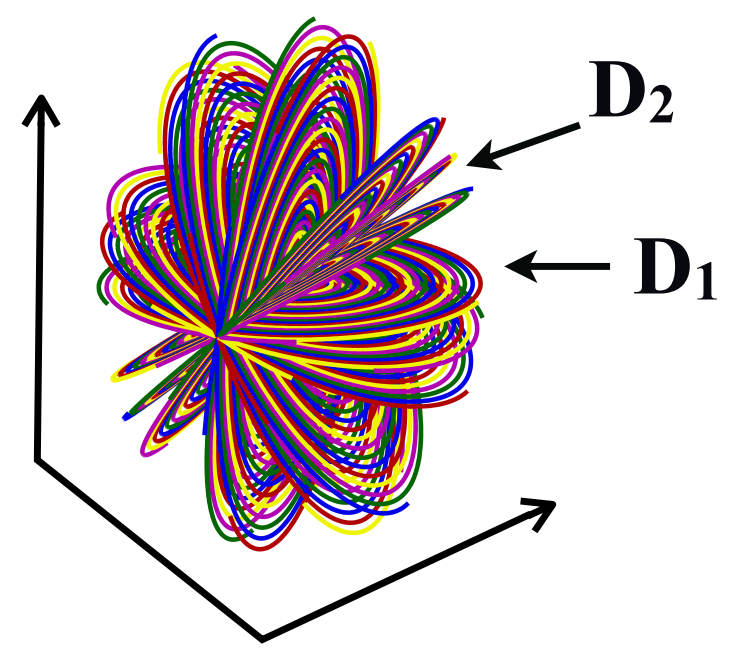


$\mathbf{D}_{1}$
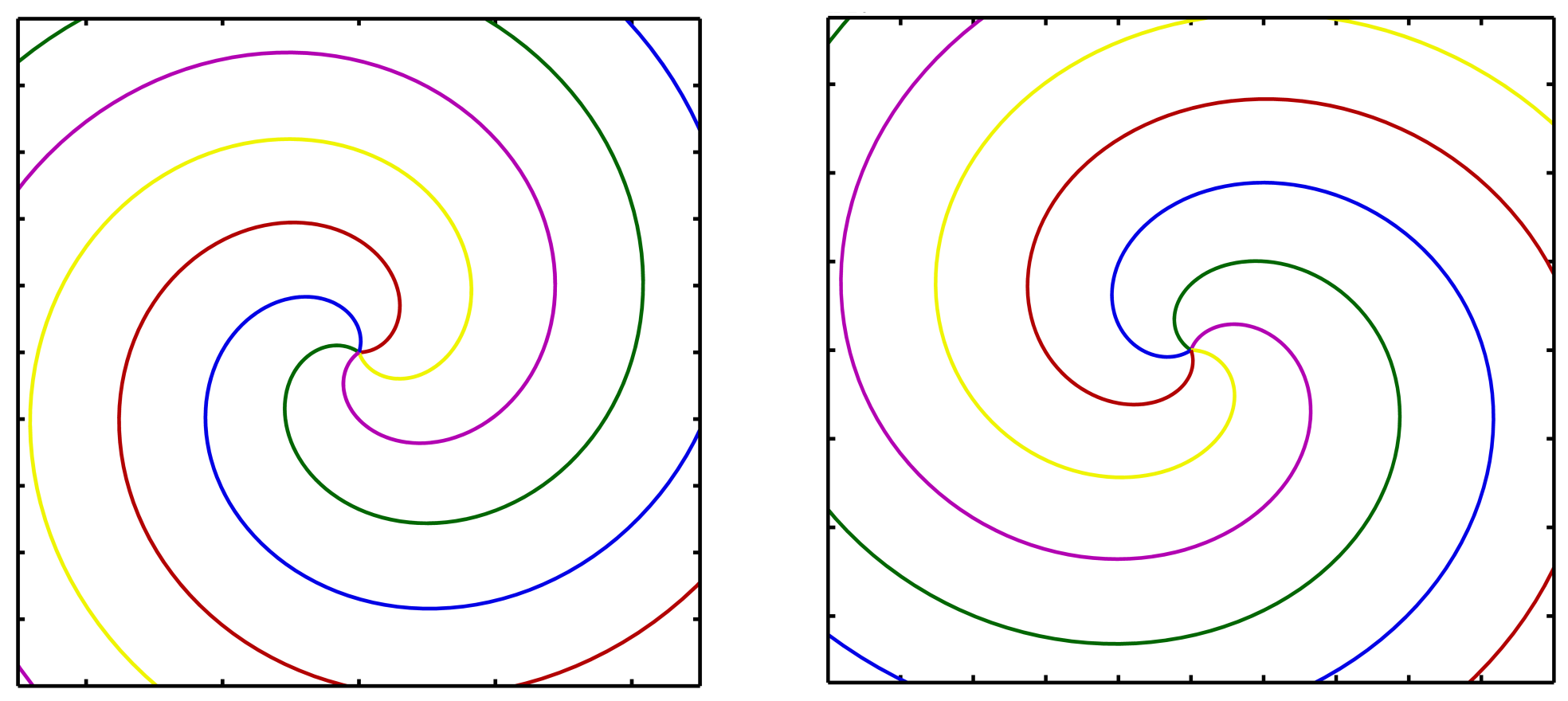

$I_{1}$, TR number $=1$

$I_{2}$, TR number $=11$

I3, TR number $=21$

$I_{4}, \mathbf{T R}$ number $=31$

I5, TR number $=41$
$\mathbf{D}_{2}$

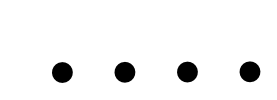

$I_{1}$, TR number $=2$

$I_{2}$, TR number $=12$

$I_{3}$, TR number $=22$

$I_{4}$, TR number $=32$

I5, TR number $=42$
$\mathbf{D}_{10}$

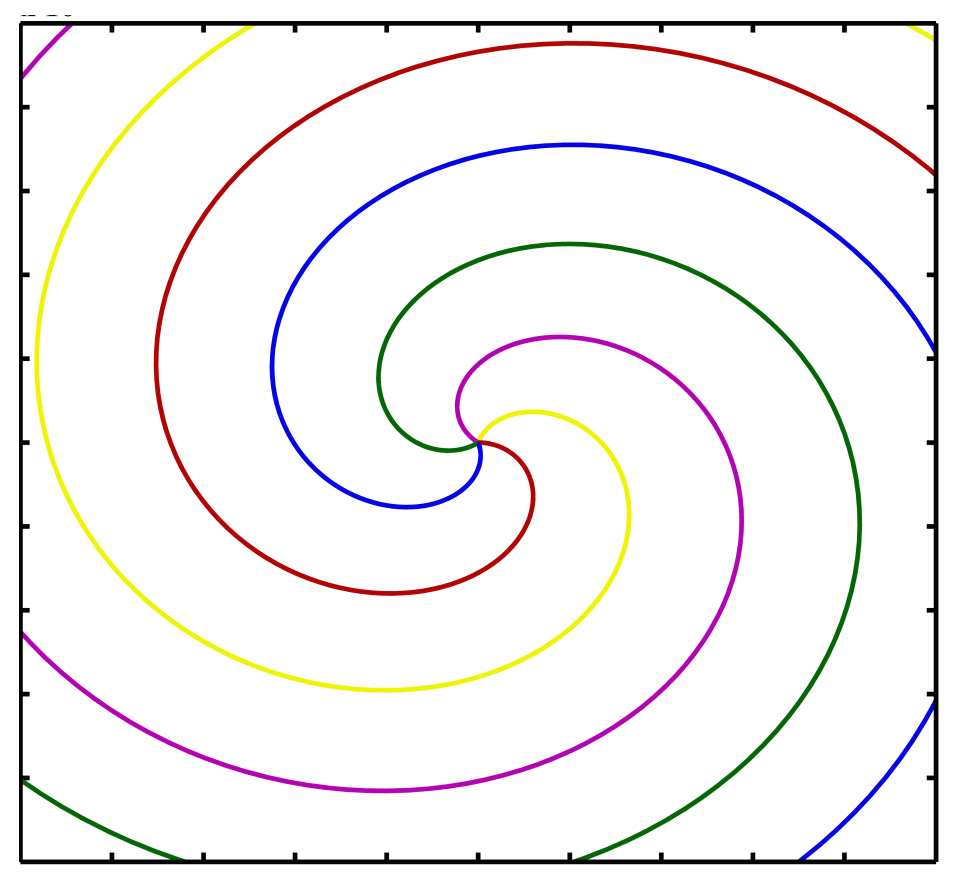

$I_{1}$, TR number $=10$

$I_{2}$, TR number $=\mathbf{2 0}$

$I_{3}$, TR number $=30$

$I_{4}$, TR number $=40$

I5, TR number $=\mathbf{5 0}$ 
Undersampling factor

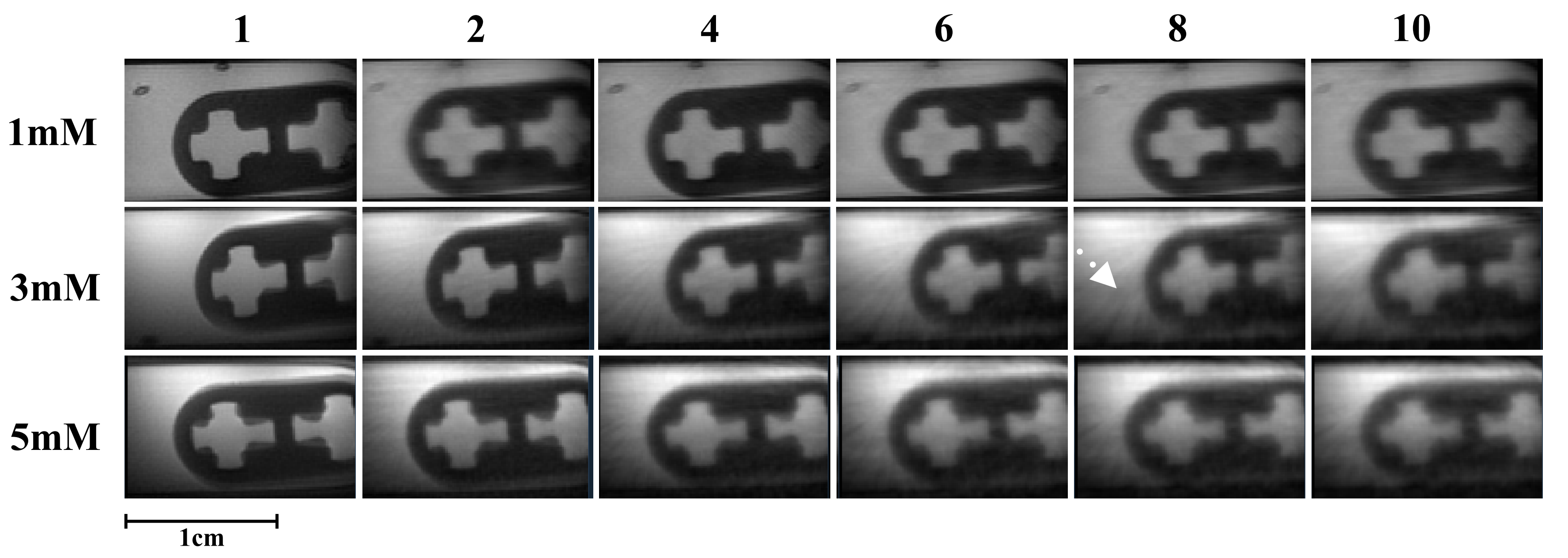


$\underline{\text { Ninterleaves } / \text { Npoints }}=\quad$ Ninterleaves $/$ Npoints $=\quad$ Ninterleaves $/$ Npoints $=$ Ninterleaves $/$ Npoints $=$

a)

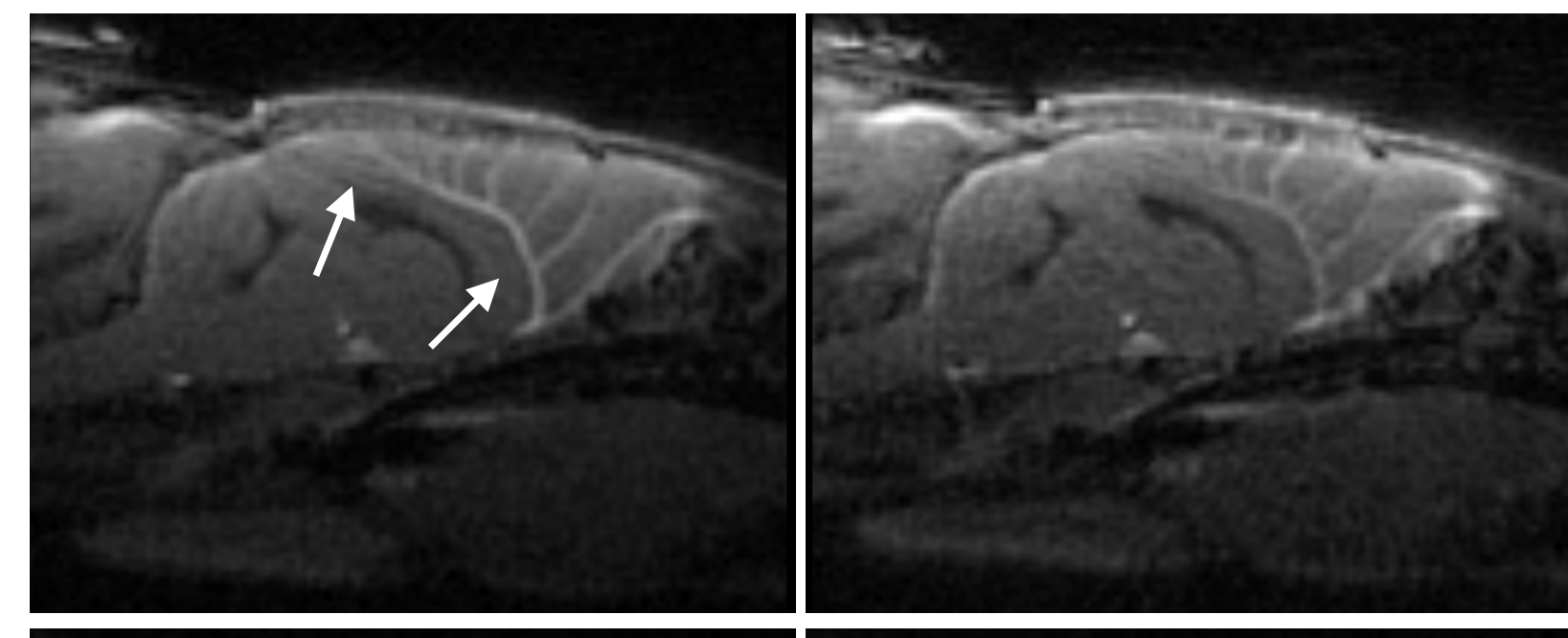

b)
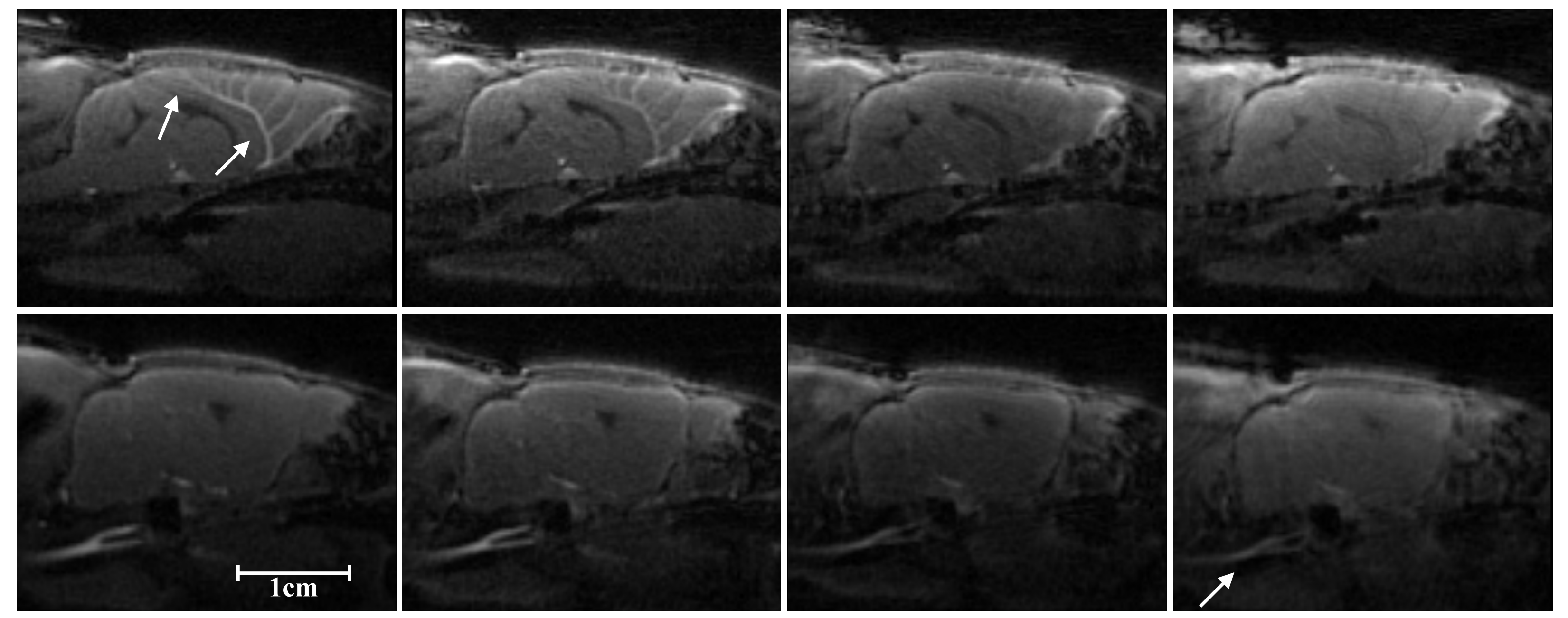


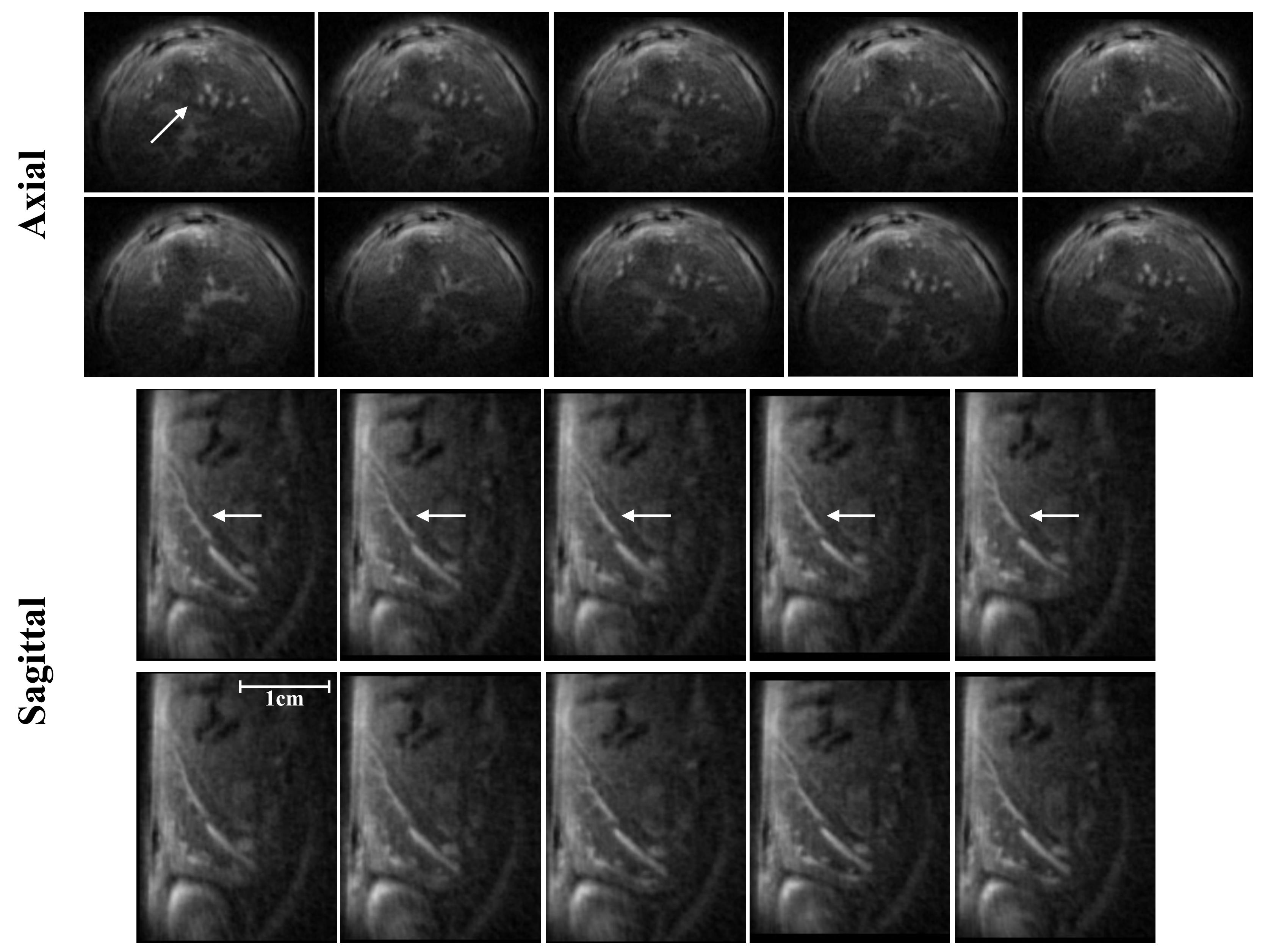

